\title{
Segmentation of melanoma images
}

\author{
Nery Javier Machado Báez José Luis Vázquez Noguera Horacio Legal Ayala \\ Polytechnic School, National University of Asuncion, \\ P.O.Box: 2111 SL, San Lorenzo, Paraguay \\ E-mail: neryjavier@gmail.com, \{jvazquez,hlegal $\} @$ pol.una.py
}

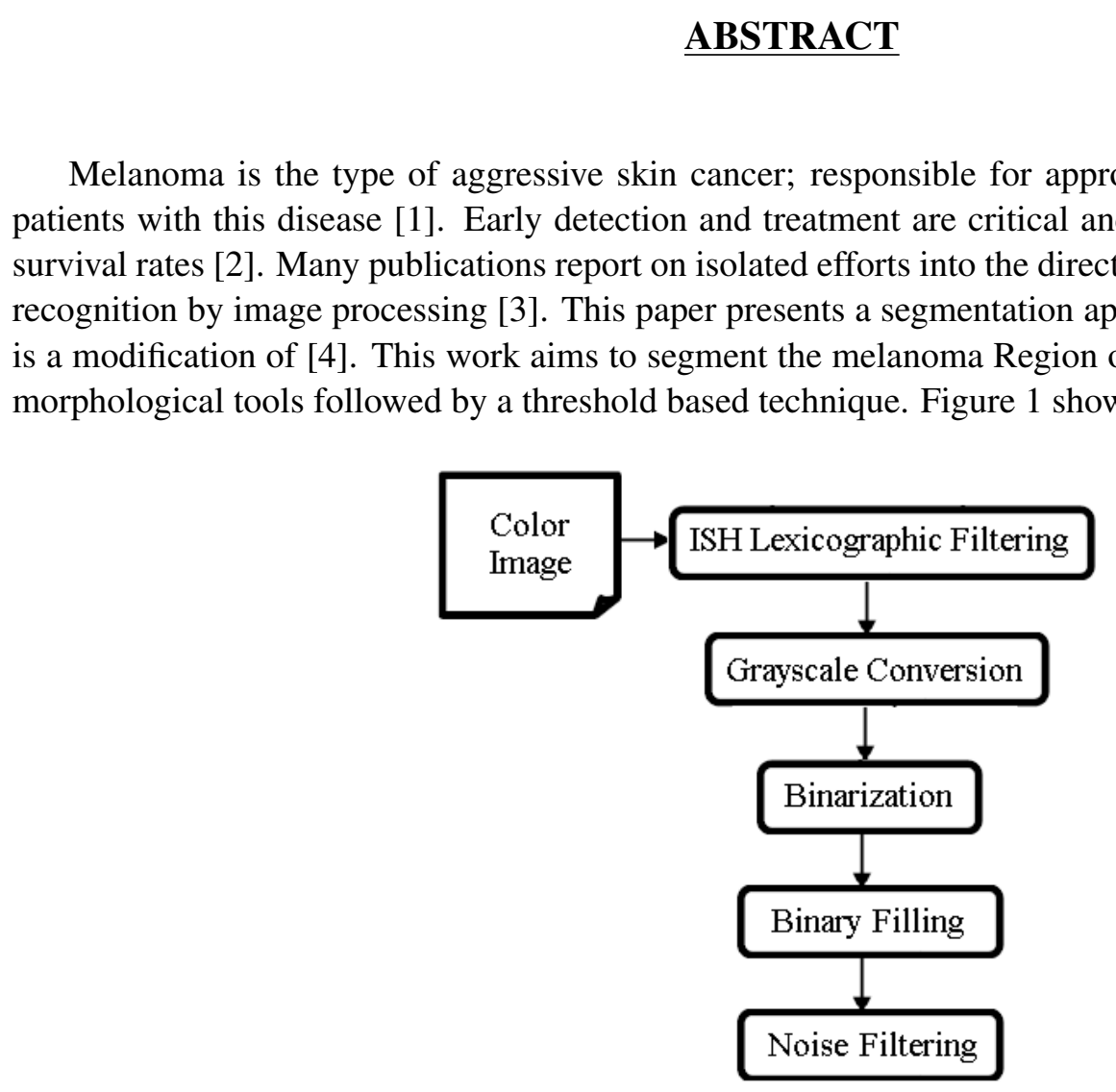

Figure 1: Flowchart of the melanoma segmentation

In [4] Beuren used the Renyi's entropy [5] as thresholding method (Binarization). In [6] there were given the different analyzes of thresholding methods on different grayscale images and channels of color; they concluded that the kittler method [7] is better in most cases comparing to other thresholding methods. For this reason, the thresholding method in [4] was changed, using the kittler method. The approach was tested on two benign and malignant image databases, both containing 100 images, and the results were compared to ground-truth segmentation and to the approach in [4]. Best results were obtained in the metrics of Precision (96\% $\pm 5 \%$ of benign and $95 \% \pm 5 \%$ of malignant segmentation) and Accuracy ( $96 \% \pm 3 \%$ of benign and $95 \% \pm 3 \%$ of malignant segmentation).

An example of the approach is depicted in Figure 2. In (a) it is shown the original image (benign), in (b) the ground truth image, in (c) the result of our proposed approach, and in (d) the result of the proposed approach in [4]. Numerical results show that image (c) is more similar to ground truth than image (d). 


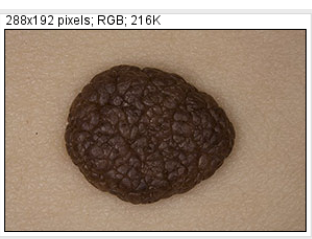

(a)

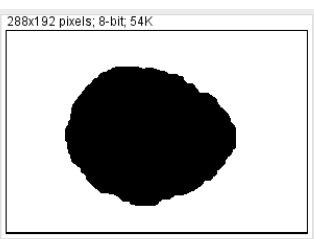

(b)

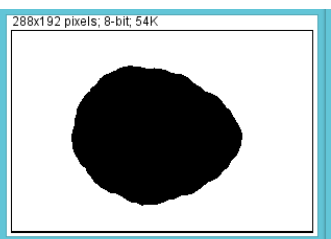

(c)

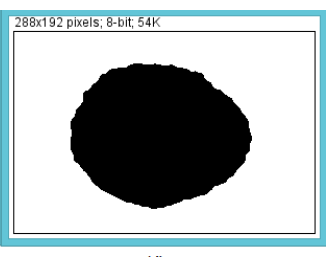

(d)

Figure 2: (a) Original image, (b) Ground truth image, (c) Our proposed approach (d) The proposed approach in [4]

Keywords: Melanoma, Binarization, Segmentation

\section{References}

[1] A. J. Swerdlow, The risk of melanoma in patients with congenital nevi: a cohort study, in "Journal of the American Academy of Dermatology", vol. 32, no. 4, pp. 595-599, 1995.

[2] D. S. Rigel, Malignant melanoma: prevention, early detection, and treatment in the 21 st century, in "CA: a cancer journal for clinicians", vol. 50, no. 4, pp 215-236, 2000.

[3] H. Ganster, et al, Automated melanoma recognition in "Medical Imaging, IEEE Transactions", vol. 20, no. 3, pp. 233-239, 2001.

[4] A. T. Beuren, R. JG Pinheiro, and J. Facon, Color approach of melanoma lesion segmentation, in "Systems, Signals and Image Processing (IWSSIP), 2012 19th International Conference on. IEEE", 2012.

[5] P. Sahoo, C. Wilkins, and J. Yeager, Threshold selection using Renyi's entropy in "Pattern recognition"”, vol.30 no.1, pp. 71-84, 1995.

[6] C. A. Cattaneo, L. I. Larcher, A. I. Ruggeri, A. C. Herrera, E. Biasoni and M. Escauelas, Métodos de umbralizacin de imágenes digitales basados en entropia de Shannon y otros in "Mecánica Computacional Vol XXX”, pp. 2785-2805, 2011.

[7] J. Kittler, J. Illingwortj. Minimum error thresholding in "Pattern recognition", vol. 19 no. 1, pp. 41-47, 1986. 